\title{
PERFORMANCE DE PLANTAS LEGUMINOSAS PARA SISTEMA PLANTIO DIRETO NA REGIÃO AMAZÔNICA
}

\author{
Andréia Marcilane Aker ${ }^{1}$, Alexandre Martins Abdão dos Passos ${ }^{2}$ \\ ${ }^{1}$ Doutoranda em Produção Vegetal, Universidade Federal dos Vales do \\ Jequitinhonha e Mucuri - UFVJM, Diamantina, MG, Brasil \\ ${ }^{2}$ Pesquisador da Embrapa Milho e Sorgo (alexandre.abdao@embrapa.br), Sete \\ Lagoas, MG, Brasil \\ Recebido em: 22/09/2018 - Aprovado em: 23/11/2018 - Publicado em: 03/12/2018 \\ DOI: 10.18677/EnciBio_2018B66
}

\begin{abstract}
A inserção de plantas leguminosas pode representar uma estratégia para fertilização em solos distróficos na região amazônica. Objetivou-se avaliar o acúmulo de nutrientes, recalcitrância da palhada e o efeito de diversas plantas leguminosas sobre o status nutricional do milho em sucessão. Foram avaliados os teores e acúmulo de $\mathrm{P}, \mathrm{K}, \mathrm{Mg}, \mathrm{S}, \mathrm{Ca}, \mathrm{Zn}, \mathrm{Mn}$, Fe e $\mathrm{Cu}$ e a dinâmica de degradação da palhada das plantas de cobertura sobre os teores foliares dos nutrientes no milho safra semeado em sucessão às leguminosas. Observou-se que os maiores incrementos no aporte de nutrientes ocorreram na biomassa do feijão guandu e feijão de porco, na ordem de $557 \%$ e $490 \%$ respectivamente, no acúmulo de fósforo quando comparados com o tratamento mantido em pousio. A utilização de feijão de porco e crotalária juncea ocasionou incrementos médios de 12,5 e 20,8\% nos teores de magnésio foliar no milho em relação ao pousio. Avaliando-se os micronutrientes, observou-se incrementos de 14,02 e 8,49\% nos teores de manganês pelo uso da Crotalaria spectabilis e mucuna cinza respectivamente em relação à prática do pousio. A maior recalcitrância de palhada foi observada para a Crotalaria ochroleuca que promoveu um tempo de meia de vida de 148 dias, suficiente para proteção do solo durante o período de entressafra. As plantas de cobertura promovem um incremento na ciclagem de nutrientes superior à prática de pousio na região, representando uma estratégia de intensificação sustentável do uso do solo.
\end{abstract}

RESUMO

PALAVRAS-CHAVE: agricultura conservacionista, nutrição mineral de plantas, palhada

\section{PERFORMANCE OF LEGUME COVER CROPS FOR NO-TILLAGE SYSTEM IN THE AMAZON REGION}

\begin{abstract}
The use of cover crops represents an alternative to increase the fertility of the dystrophic soils in the Amazon. The aim of this paper was to evaluate the nutrients pool on the legumes cover crops shoots, the recalcitrance of their straw and the influence of the cover crops on the nutritional status of maize plants. The contents of $\mathrm{P}, \mathrm{K}, \mathrm{Mg}, \mathrm{S}, \mathrm{Ca}, \mathrm{Zn}, \mathrm{Mn}, \mathrm{Fe}$ and $\mathrm{Cu}$ on the cover crops, and the dynamics of straw degradation of legumes plants in corn plants succession to legumes were evaluated. It was observed that the highest level of $P$ on the legume's biomass was in the pigeon pea and jack bean. These plants present 557\% and 490\% more $P$ in these
\end{abstract}


tissue in relation to the fallow treatment. The use of jack bean and crotalaria juncea boosted in 12.5 and $20.8 \%$ corn foliar magnesium in relation to fallow. The micronutrients level in the corn were evaluated, and it was observed increments of 14.02 and $8.49 \%$ in the manganese contents by the use of Crotalaria spectabilis and in the grey mucuna in relation to the fallow. The greatest recalcitrant straw was observed for the crotalaria ochroleuca that promoted a life time of 148 days, sufficient for the protection of the soil during the off-season. As the cover plants promote a higher nutrition increment on corn plants than the traditional fallow practice in the region, they are an option of sustainable intensification of land use in Amazonia.

KEYWORDS: conservation agriculture, mineral nutrition of plants, straw

\section{INTRODUÇÃO}

No processo de implementação ou durante a manutenção do sistema de plantio direto, a cobertura vegetal viva e morta em forma de palhada promove o incremento no sistema, resultando no sucesso da lavoura (DERPSCH et al., 2014). Quando inseridas apropriadamente as plantas de cobertura mantêm a camada superficial do solo continuamente coberto de biomassa vegetal, reciclam nutrientes tornando-os gradualmente disponíveis para cultivos sucessivos (AKER et al., 2016). Dessa forma, ao se decompor, os resíduos das culturas são vistos como uma fonte de nutrientes e o conhecimento de sua dinâmica de degradação é fundamental para a escolha da espécie adequada a ser inserida no sistema (XAVIER et al., 2017).

O equilíbrio entre a vegetação e o solo é essencial para a manutenção de sua fertilidade, o que possibilita a adequada ciclagem dos nutrientes através da decomposição de detritos orgânicos e dos processos biogeoquímicos, como por exemplo, na fixação biológica do nitrogênio (MBUTHIA et al., 2015). O acúmulo de biomassa sobre o solo, em forma de palhada, promove um microambiente estável, de temperaturas mais amenas e maior umidade, que favorece as atividades biológicas que auxiliam no processo de decomposição da palhada e na disponibilidade e absorção dos nutrientes pelas culturas (SANTOS et al., 2010). O acúmulo de matéria orgânica do solo produzidos pela cobertura vegetal pode representar ainda uma estratégia de mitigação de emissões de gases de efeito estufa (LAL, 2015).

A permanência da cobertura vegetal sobre o solo depende da taxa de decomposição (CAVALLI et al., 2018), que varia em função da espécie cultivada, bem como de sua composição química (VALADARES et al., 2012), além dos fatores climáticos (MIRANDA et al., 2018), da biomassa inicial e idade do vegetal na época do manejo (CARVALHO et al., 2015) e da forma de manejo adotado. Dessa forma, a quantidade e a qualidade de resíduos produzidos dependem da escolha adequada das plantas de coberturas (TORRES et al., 2015), colaborando para o aumento da produção e recuperação ou manutenção da qualidade do solo (DARYANTO et al., 2018).

Em regiões de clima tropical, as condições de altas temperaturas e precipitação, são propícias aos processos de degradação do solo, principalmente pela perda de solo e nutrientes pela erosão. Na Amazônia, ainda são escassas as pesquisas que indiquem espécies apropriadas para compor a palhada para sistemas plantio direto. Neste sentido, o objetivo desta pesquisa foi de avaliar o acúmulo de nutrientes e a dinâmica da palhada de plantas de cobertura do solo em sistema de sucessão de cultura ao milho na região sudoeste da Amazônia. 


\section{MATERIAL E MÉTODOS}

O estudo foi desenvolvido na área experimental da Embrapa em Porto Velho, Rondônia, localizada a uma latitude de $8^{\circ} 47^{\prime} 53,00^{\prime \prime} \mathrm{S}$, e longitude $63^{\circ} 51^{\prime} 02,82^{\prime \prime} \mathrm{O}$ e uma altitude média de 87 metros, conduzido entre os meses de abril de 2014 a fevereiro de 2015. Em área conduzida sob plantio direto desde 2008, com a cultura de soja. Em sua classificação, o solo é considerado como Latossolo VermelhoAmarelo distrófico. Antes da instalação do experimento, foi coletada amostras de solo na camada de $0-20 \mathrm{~cm}$ de profundidade para análise para caracterização dos atributos químicos, conforme demonstrado na Tabela 01.

TABELA 01. Resultados da análise química de solo na profundidade de 0-20 avaliados em julho de 2014, Porto Velho, Rondônia.

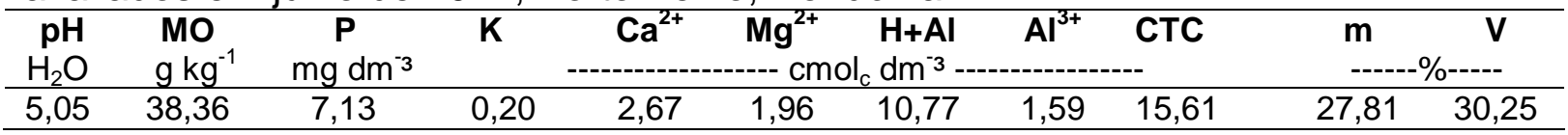

$\mathrm{pH}$ em água 1:2,5, M.O. por digestão úmida, $\mathrm{P}$ e K determinados pelo método Mehlich I, Ca, Mg e Al trocáveis extraídos com $\mathrm{KCl} 1 \mathrm{~mol} \mathrm{~L}^{-1}$, CTC: Capacidade de Troca de Cátions. m: saturação por alumínio. V: saturação por bases.

O clima da região é Am, conforme classificação de Köppen. A média anual de temperatura é em torno de $25,6^{\circ} \mathrm{C}$ e precipitação média anual é de $2.200 \mathrm{~mm}$, possui uma estação chuvosa definida que se estende de outubro a maio, enquanto a estação seca vai de junho a setembro com evapotranspiração potencial média anual de $1.455 \mathrm{~mm}$ (ALVARES et al., 2013). Na Figura 01, se observam os dados climatológicos coletados durante o período experimental.

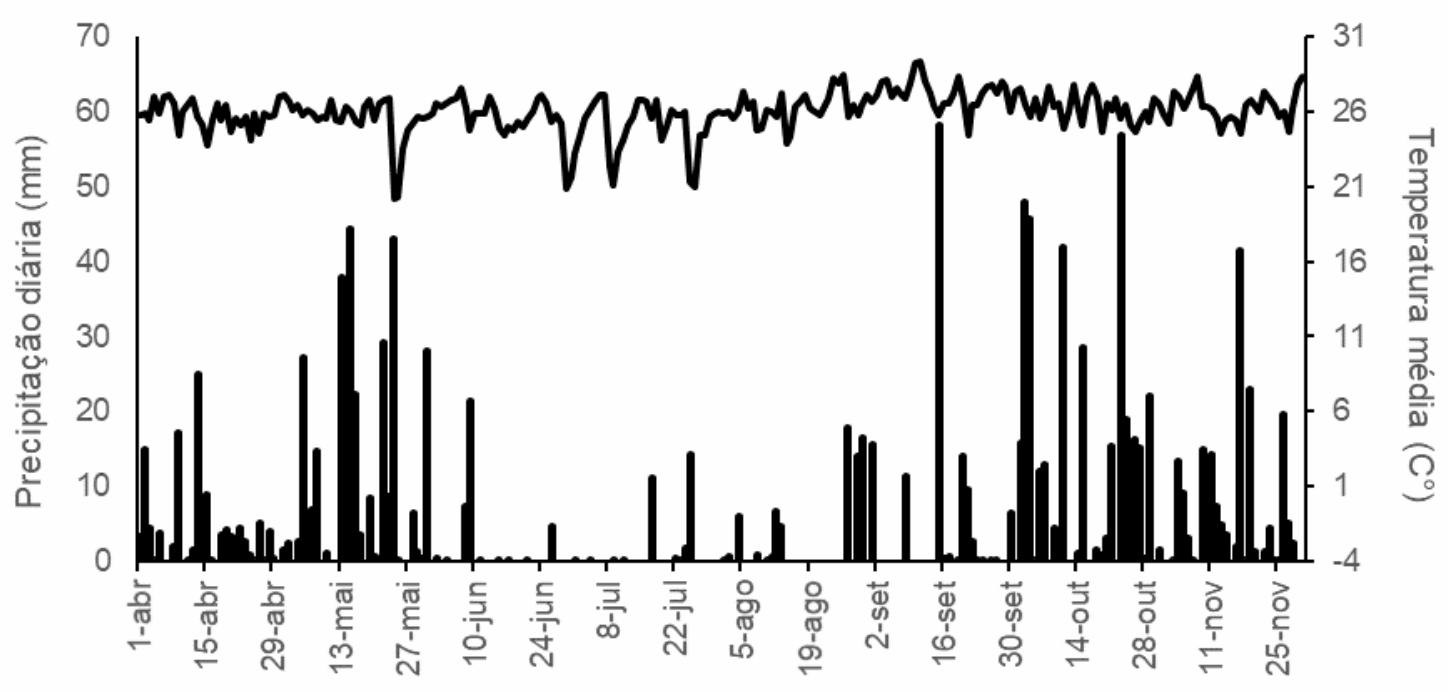

FIGURA 01 - Precipitação pluviométrica (barras) e temperatura diária média (linha) no período de abril a novembro de 2014, em Porto Velho, Rondônia. Fonte: Instituto Nacional de Meteorologia, 2014.

Os tratamentos correspondem ao pousio mais 7 plantas de cobertura leguminosas em sucessão à soja na safra 2013/2014, a saber: Crotalaria juncea; Crotalaria ochroleuca; Crotalaria spectabilis; Feijão guandú (Cajanus cajan); Feijão de porco (Canavalia ensiforme); Mucuna cinza (Stizolobium cinereum) e Mucuna preta (Stizolobium aterrina). O experimento foi realizado com delineamento em 
blocos casualizados com quatro repetições. Cada parcela referente à uma cobertura de solo constituiu-se de $50 \mathrm{~m}^{2}(5 \mathrm{~m} \times 10 \mathrm{~m})$. A semeadura das plantas leguminosas ocorreu de forma manual em campo em abril de 2014 durante a entressafra do milho verão. A abertura dos sulcos foi realizada mecanicamente com semeadora, adotando-se um espaçamento de 0,45 metros. Não se realizou inoculação das sementes e adubação de semeadura.

As espécies leguminosas foram manejadas por meio de corte com roçadeira costal em julho de 2014, enquanto estavam no florescimento (estádio fenológico definido a partir de ao menos $50 \%$ das plantas da parcela em florescimento). Em seguida aos cortes, foi retirado o material cortado da parte aérea de uma área representativa de $0,25 \mathrm{~m}^{2}$ em dois pontos amostrais de cada parcela, sendo aferida a produção de massa verde e posteriormente determinada a massa da matéria seca em estufa com ventilação forçada de ar a $65^{\circ} \mathrm{C}$ por 72 horas.

Dessas amostras, foram retiradas alíquotas que foram triturados em moinho tipo Willey (malha de $2 \mathrm{~mm}$ ), para análise química do tecido vegetal para determinação dos teores dos macronutrientes (fosforo - $\mathrm{P}$, potássio - K, cálcio - $\mathrm{Ca}$, magnésio - Mg e enxofre - S) e micronutrientes (cobre - Cu, ferro - Fe, manganês Mn e zinco - Zn) da parte aérea, segundo metodologia descrita por Malavolta et al. (1997). Com base nos valores obtidos, foi calculado o acúmulo de cada nutriente presente na parte aérea das plantas de cobertura, por meio da relação do teor do nutriente pela matéria seca de cada parte.

Para avaliação da taxa de decomposição, foram utilizadas bolsas de decomposição (litter bags), conforme descrito por Santos e Whilford, (1981) e Boer et al. (2007). As bolsas foram confeccionadas com telas de náilon, com malha de 2 $\mathrm{mm}$. As dimensões das bolsas foram de 0,2 $\times 0,2 \mathrm{~m}$. A quantidades das palhadas utilizadas para preenchimento das bolsas foi calculada proporcionalmente entre a quantidade de massa produzida por hectare de cada espécie e a área da bolsa. A palhada acondicionada no interior dos litter bags foi distribuída aleatoriamente em campo de suas respectivas parcelas experimentais, constituindo a biomassa da matéria seca inicial de cada alternativa de cobertura do solo. Foram realizadas cinco coletas das bolsas, aos 8, 15, 60, 75 e 125 dias após o corte (DAC) para determinação da curva e parâmetros de degradação da palhada. A cada período de amostragem, os resíduos contidos nas bolsas foram secos em estufa de circulação forçada de ar, a $65{ }^{\circ} \mathrm{C}$, até atingirem peso constante, para determinação da massa da matéria seca remanescente. Aos resultados da biomassa remanescente nos litter bags, foi ajustado o modelo exponencial, com a percentagem de resíduo remanescente em função do tempo: eq. (1)

$$
X=X_{0} e^{-k t}(1)
$$

Sendo $X$ o percentual de matéria seca remanescente após um período de tempo (t) em dias; $X_{0}$ o percentual de massa da matéria seca inicial e $k$ a constante de decomposição do resíduo. Com base na constante de decomposição (k) da equação exponencial, calculou-se o tempo de ciclagem do material vegetal: eq. (2) que corresponde ao período (dias) de tempo necessário para que a metade dos resíduos se decomponha (THOMAS; ASAKAWA, 1993; TORRES et al., 2015).

$$
\mathrm{T} 1 / 2=0,69315 / \mathrm{k}(2)
$$

Em seguida, sobre as palhadas das plantas de cobertura semeadas em abril de 2014, realizou-se a semeadura do milho (hibrido simples RB 9308 YG VTPRO) no dia 03/12/2014, com o auxílio de uma semeadora-adubadora, adotando um espaçamento de $0,90 \mathrm{~m}$ e uma população final de plantas de 70.000 plantas ha ${ }^{-1}$. 
Para a adubação de semeadura, foi utilizada a fórmula NPK 05-25-20 na dose de $400 \mathrm{~kg} \mathrm{ha}^{-1}$. Realizou-se adubação de cobertura, utilizando $140 \mathrm{~kg} \mathrm{ha}^{-1}$ de nitrogênio, nos estádios fenológicos V4 (70 kg ha ${ }^{-1}$ na forma de sulfato de amônio) e V8 (70 kg $\mathrm{ha}^{-1}$ na forma de ureia), ambas foram distribuídas manualmente em filetes paralelo as plantas. Todos os demais tratos culturais foram realizados conforme preconizado para a cultura, não havendo necessidade de controle de insetos pragas e ervas daninhas na área.

Quando as plantas atingiram o estádio R1 (pleno florescimento feminino), foram coletadas folhas para análise foliar dos teores de macro e micronutrientes. As amostras foliares foram secas em estufa de circulação forçada de ar, a $65^{\circ} \mathrm{C}$, até massa constante, moídas em moinho tipo Willey e submetidos a análise do material vegetal (MALAVOLTA et al., 1997).

As médias obtidas, garantidos os pressupostos de normalidade $\mathrm{e}$ homocedasticidade para os erros, foram submetidas ao teste de F. Quando pertinente, devido à significância dos tratamentos, foi realizado teste de Scott-Knott para a comparação das médias de acúmulo e teores de nutrientes. Para o estudo da degradação de palhada, utilizou-se regressões em esquema de parcelas subdivididas no tempo. Foi utilizado para as análises o programa estatístico SISVAR.

\section{RESULTADOS E DISCUSSÃO}

Os diferentes sistemas de sucessão de cultura diferiram entre si quanto ao acúmulo e teores de macro e micronutrientes avaliados na biomassa seca das plantas leguminosas (Tabela 2). Todas as espécies apresentaram acúmulo de nutrientes superior ao pousio para fósforo, cálcio, enxofre, cobre e ferro. Havendo ao menos duas plantas de cobertura (feijão de porco e feijão guandu) que diferiram em todos os elementos.

TABELA 02 - Acúmulo de macronutrientes e micronutrientes contido no tecido vegetal das plantas de cobertura na ocasião do corte, Porto Velho, Rondônia, 2014.

\begin{tabular}{|c|c|c|c|c|c|c|c|c|c|c|c|c|c|c|c|c|c|}
\hline \multirow[t]{2}{*}{ Sistemas } & $\mathbf{P}$ & & K & & $\mathrm{Ca}$ & & $\mathbf{M g}$ & & $\mathbf{s}$ & & $\mathrm{Cu}$ & & $\mathrm{Fe}$ & $\mathbf{M n}$ & & $\mathrm{Zn}$ & \\
\hline & \multicolumn{17}{|c|}{$\mathrm{kg} \mathrm{ha}^{-1}$} \\
\hline Feijão de porco & 27,9 & a & 251,4 & a & 191,8 & $\mathrm{a}$ & 51,4 & a & 27,3 & A & 70,7 & b & $3955,0 \mathrm{~b}$ & b 615,5 & a & 224,2 & a \\
\hline Guandu & 25,1 & $\mathrm{a}$ & 161,2 & $b$ & 60,8 & c & 32,4 & $b$ & 19,2 & B & 108,5 & $a$ & $4935,5 \mathrm{~b}$ & b 502,2 & a & 295,8 & a \\
\hline C. spectabilis & 20,5 & $b$ & 175,3 & $b$ & 135,2 & $\mathrm{~b}$ & 40,0 & $\mathrm{~b}$ & 19,0 & $B$ & 116,7 & a & 2314,7 b & b 367,7 & $b$ & 292,7 & a \\
\hline C. ochroleuca & 19,9 & b & 158,1 & $\mathrm{~b}$ & 48,5 & c & 37,6 & $\mathrm{~b}$ & 18,0 & B & 81,7 & a & $1147,9 \mathrm{~b}$ & b 221,1 & $\mathrm{~b}$ & 271,0 & $\mathrm{a}$ \\
\hline Mucun & 19,8 & $\mathrm{~b}$ & 114,4 & c & 41,6 & c & 17,9 & d & 12,2 & C & 97,2 & a & $1322,5 \mathrm{~b}$ & b 215,5 & $\mathrm{~b}$ & 187,6 & $\mathrm{~b}$ \\
\hline Mucuna cinza & 16,0 & c & 92,5 & c & 52,3 & c & 19,4 & $d$ & 11,8 & $\mathrm{C}$ & 107,3 & a & $1675,3 \mathrm{~b}$ & b 373,8 & $b$ & 165,6 & $b$ \\
\hline Crotalaria juncea & 13,7 & c & 98,9 & c & 45,9 & c & 27,9 & $d$ & 10,1 & C & 63,6 & $b$ & $1430,1 \mathrm{~b}$ & 199,5 & $b$ & 133,3 & $b$ \\
\hline Pousio & 4,3 & d & 52,7 & c & 11,2 & $d$ & 10,2 & d & 3,6 & D & 16,2 & c & 13207,4 & a 222,5 & $\mathrm{~b}$ & 46,8 & $\mathrm{~b}$ \\
\hline
\end{tabular}

Médias seguidas pela mesma letra, na coluna, não diferem entre si ao nível de $5 \%$ de significância pelo teste de Scott-knott $(p<0,05)$.

Observa-se no cultivo de feijão de porco os maiores incrementos em todos os macronutrientes avaliados. Resultados semelhantes foram encontrados por Heinrichs et al. (2005), na qual, o feijão de porco teve os maiores rendimentos nos acúmulos de $\mathrm{N}, \mathrm{P}, \mathrm{K}, \mathrm{Ca}, \mathrm{Mg}$ e $\mathrm{S}$ em relação ao cultivo de mucuna anã, guandu anão, crotalária e pousio. Maiores acúmulos de nutrientes podem representar reflexos positivos sobre o rendimento de grãos em solos distróficos da Amazônia (AKER et al., 2016). 
Os estoques de $\mathrm{P}$ na fitomassa das plantas de cobertura variaram de 4,25 a $27,92 \mathrm{~kg} \mathrm{ha}^{-1}$ (Tabela 02). As culturas de feijão de porco e guandu tiveram as maiores capacidades de acúmulo, apresentando um incremento de $557 \%$ e $490 \%$ respectivamente, quando comparados com o tratamento controle (solo mantido em pousio). $\mathrm{O}$ uso de espécies que possuem alta capacidade de acúmulo de $\mathrm{P}$ na parte aérea, é uma alternativa para elevar a disponibilidade deste elemento para as plantas, visto que, ao serem liberadas para o solo.

Teixeira et al. (2010) constataram que o acúmulo de $\mathrm{P}$ na palhada do consórcio entre milheto e feijão-de-porco foi superior $\left(7,47 \mathrm{~kg} \mathrm{ha}^{-1}\right)$ ao uso de milheto solteiro $\left(3,81 \mathrm{~kg} \mathrm{ha}^{-1}\right)$, dando à palhada do consórcio maior capacidade de liberação de $\mathrm{P}$ ao longo do estudo. O feijão-de-porco apresenta vantagens por sua rusticidade, é uma cultura tolerante a altas temperaturas e à seca adaptando-se a diversos tipos de solo, inclusive em ambientes pobres em fósforo (MATATA et al., 2017).

Quanto ao acúmulo de K, deve-se escolher espécies que são mais eficientes no acúmulo deste nutriente, afim de disponibiliza-lo para a próxima cultura em sistemas de sucessão (AKER et al., 2017), visto que esse elemento pode ser facilmente extraído dos tecidos das plantas, tanto pela água da chuva quanto pela própria umidade do solo, devido a sua rápida liberação (BOER et al., 2007). O maior incremento de $\mathrm{Ca}$ promovido pelo feijão de porco pode estar relacionado a produtividade de biomassa. Estudos realizados por Pereira et al. (2017) na região Sul do Brasil com seis culturas de verão como planta de cobertura, em seus resultados teve o feijão de porco com os maiores acúmulos e enfatizam que a cultura possui a capacidade de ciclagem de nutrientes como o potássio e cálcio devido à elevada imobilização destes nutrientes na parte aérea nessa leguminosa.

Com relação aos acúmulos de micronutrientes, ocorreram diferenças entre os tratamentos (Tabela 2). Quanto ao acúmulo de $\mathrm{Cu}$, o pousio apresentou o menor valor $\left(16,17 \mathrm{~kg} \mathrm{ha}^{-1}\right)$, seguido pelas leguminosas a Crotalaria juncea $\left(63,60 \mathrm{~kg} \mathrm{ha}^{-1}\right)$ e o feijão de porco $\left(70,72 \mathrm{~kg} \mathrm{ha}^{-1}\right)$. Para o $\mathrm{Fe}$, não houve diferença entre as leguminosas avaliadas, apenas a vegetação espontânea, representada pelo pousio que apresentou a maior média. Enquanto os acúmulos de Mn sobressaíram na cultura de feijão de porco e guandu. Já para o Zn, o guandu, Crotalaria spectabilis, Crotalaria ochroleuca e o feijão de porco, não diferiram entre si, mas apresentaram média superior às mucuna preta, mucuna cinza, Crotalaria juncea e ao pousio.

Dessa forma, a prática de sucessão de culturas ou a adubação verde, principalmente com uso de leguminosas, constitui uma importante maneira de adicionar nutrientes, podendo substituir parcialmente o adubo mineral, promovendo a reciclagem de nutrientes para as plantas, com vantagens, ao promover uma liberação lenta e sincronizada, de acordo com as necessidades das plantas (SANTOS et al., 2010).

Os resultados não evidenciaram efeitos significativos para os teores foliares dos macronutrientes $\mathrm{P}, \mathrm{K}, \mathrm{Ca}$ e $\mathrm{S}$ na cultura do milho (Tabela 3). Porém, verificou-se efeito dos sistemas de culturas sobre os teores de $\mathrm{Mg}$, no tecido foliar do milho, sendo que os maiores teores foram obtidos no sistema após o uso de Crotalaria juncea $\left(2,91 \mathrm{~g} \mathrm{~kg}^{-1}\right)$ e de feijão de porco $\left(2,68 \mathrm{~g} \mathrm{~kg}^{-1}\right)$. O magnésio é um nutriente essencial para a eficiência fotossintética dos vegetais, como fundamental componente da clorofila, sobretudo do milho. Por ser uma planta C4 sua demanda por energia metabólica é superior à requerida por plantas de metabolismo C3. Dessa forma, pode ocorrer redução em produtividade em sistemas que apresentem menores teores de $\mathrm{Mg}$, quando em cultivos sequentes a maior deficiência desse nutriente pode comprometer o rendimento das lavouras (VALADARES et al., 2012). 
TABELA 03 - Status nutricional por meio de teores de macronutrientes $\left(\mathrm{g} \mathrm{kg}^{-1}\right) \mathrm{e}$ micronutrientes $\left(\mathrm{mg} \mathrm{kg}^{-1}\right)$ da parte aérea do milho, Porto Velho, Rondônia, 2015.

\begin{tabular}{|c|c|c|c|c|c|c|c|c|c|c|c|c|c|c|c|c|}
\hline \multirow[t]{2}{*}{ Sistemas } & $\mathbf{P}$ & & K & & $\mathrm{Ca}$ & Mg & & \multicolumn{2}{|l|}{$\mathbf{S}$} & $\mathrm{Cu}$ & & $\mathrm{Fe}$ & Mn & & \multicolumn{2}{|l|}{$\mathrm{Zn}$} \\
\hline & \multicolumn{6}{|c|}{$\mathrm{g} \mathrm{kg}^{-1}$} & & & & \multicolumn{7}{|c|}{$\mathrm{mg} \mathrm{kg}^{-1}$} \\
\hline Feijão de porco & 4,1 & a & 23,4 & a 2 & $2,2 \mathrm{a}$ & 2,7 & a & 2,8 & a & 10,5 & a & 179,5 & a 25,8 & b & 14,0 & \\
\hline Guandu & 4,1 & a & 20,4 & a 2 & 2,1 a & 2,5 & $b$ & 2,6 & a & 11,0 & a & 130,0 & a 28,2 & $b$ & 13,9 & a \\
\hline Crotalaria spectabilis & 4,4 & $\mathrm{a}$ & 23,5 & a 2 & $2,2 \mathrm{a}$ & 2,5 & $b$ & 2,8 & a & 11,5 & a & 128,3 & a 30,9 & a & 15,8 & $\mathrm{a}$ \\
\hline Crotalaria ochroleuca & 3,9 & a & 20,2 & a 2 & 2,2 a & 2,5 & $b$ & 2,7 & a & 10,9 & a & 150,3 & a 27,8 & b & 13,5 & a \\
\hline Mucuna preta & 3,7 & a & 21,2 & a 1 & $1,9 \mathrm{a}$ & 2,3 & $b$ & 2,4 & a & 10,2 & a & 130,6 & a 24,9 & b & 14,1 & a \\
\hline Mucuna cinza & 4,2 & $a$ & 21,9 & a 2 & $2,1 \quad a$ & 2,4 & $b$ & 2,8 & a & 11,8 & a & 129,5 & a 29,4 & $a$ & 14,9 & a \\
\hline Crotalaria juncea & 4,1 & a & 19,8 & a 2 & $2,3 a$ & 2,9 & a & 2,7 & a & 11,2 & a & 155,6 & a 26,8 & $\mathrm{~b}$ & 15,4 & $a$ \\
\hline Pousio & 4,1 & a & 20,7 & a 2 & $2,1 \mathrm{a}$ & 2,4 & $b$ & 2,5 & a & 10,4 & a & 123,1 & a 27,1 & $\mathrm{~b}$ & 14,0 & $\mathrm{a}$ \\
\hline
\end{tabular}

Médias seguidas pela mesma letra não diferem entre si ao nível de $5 \%$ de significância pelo teste de Scott-knott $(p<0,05)$.

Quanto aos micronutrientes avaliados, apenas o $\mathrm{Mn}$ influenciou significativamente o status nutricional do milho (Tabela 3 ), tendo como destaque as culturas Crotalaria spectabilis (30, $\left.94 \mathrm{mg} \mathrm{kg}^{-1}\right)$ e mucuna cinza $\left(29,44 \mathrm{mg} \mathrm{kg}^{-1}\right)$.

As plantas de cobertura apresentaram diferentes comportamentos quanto à persistência no solo (Figura 2 e Tabela 4). Conhecer a dinâmica de decomposição de diferentes plantas de coberturas e manejo da palhada das mesmas, dentro do sistema de plantio direto, é imprescindível para que se tenha uma gama de espécies a serem indicadas e utilizadas nas rotações, buscando produzir quantidade adequada de massa seca e liberação de nutrientes para a cultura em sucessão (TEIXEIRA et al., 2010).

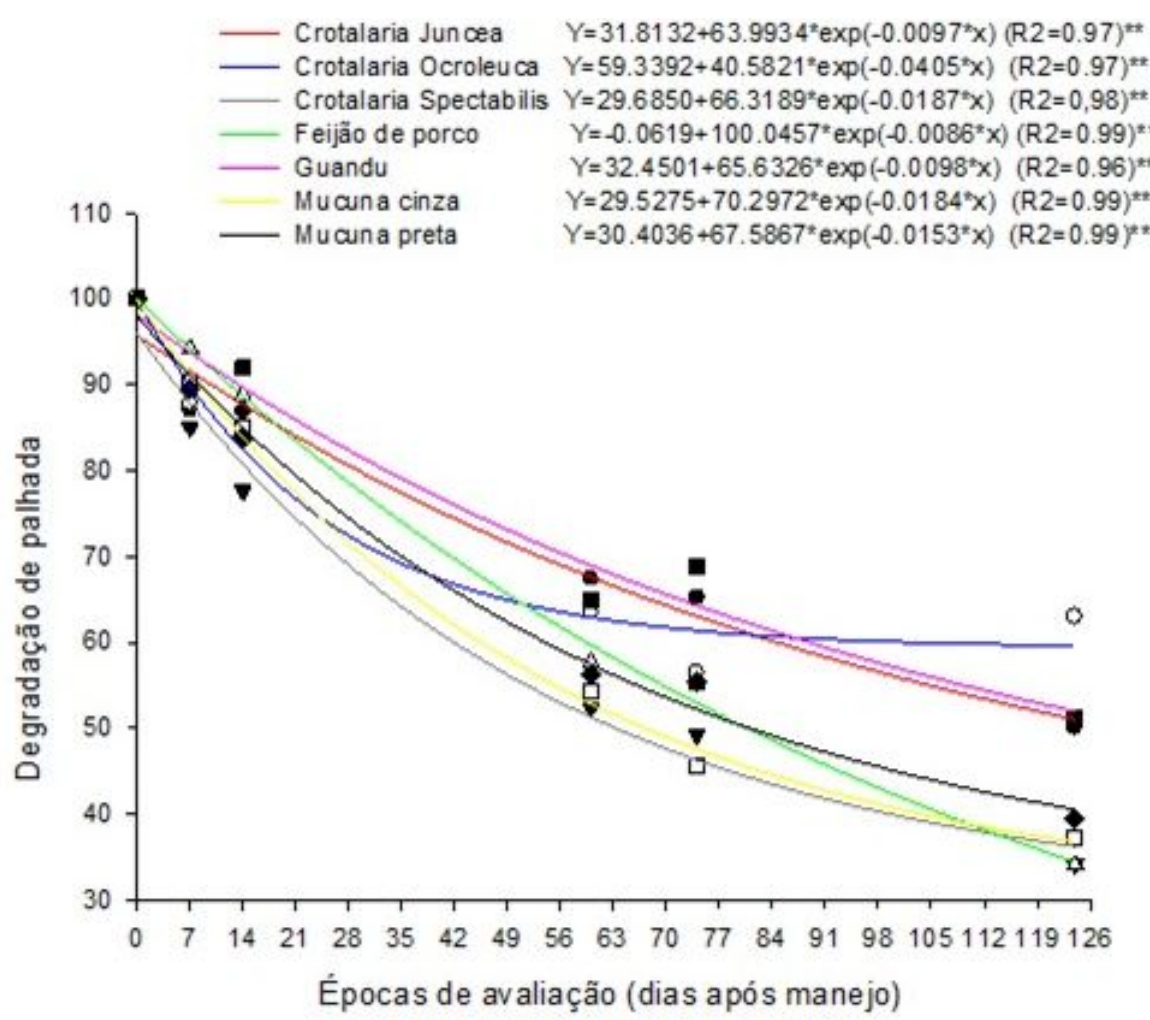

FIGURA 02 - Massa de matéria seca remanescente, em porcentagem, das espécies utilizada para cobertura do solo, Porto Velho, Rondônia, 2014.

Fonte: Autores 
De modo geral, as leguminosas apresentaram, em média, permanência sobre o solo superior ao período de entressafra, que corresponde aproximadamente aos 4 meses entre a época de corte das plantas e a época de semeadura das culturas de safra, que na região inicia-se em meados de outubro.

As leguminosas Crotalaria ochroleuca, Crotalaria juncea e o feijão guandú apresentaram persistência de palhada compatível com o esperado para plantas de cobertura para a região (Tabela 4). Estas espécies apresentaram tempos de meia vida superior ao período de entressafra e de maior estresse hídrico ocorrente na região de 120 dias (ALVARES et al., 2013).

TABELA 4 - Parâmetros de decomposição ( $\mathrm{k}=$ constante de degradação), tempo de meia-vida $\left(T^{1} 12\right)$ da massa da matéria seca remanescente derivada de plantas de cobertura leguminosas na região sudoeste da Amazônia. 2015.

\begin{tabular}{lccc}
\hline $\begin{array}{l}\text { Sistema de } \\
\text { Sucessão }\end{array}$ & $\mathrm{g} \mathrm{g}^{-1}$ & $\begin{array}{c}\mathrm{T} 1 / 2 \\
\text { (dias) }\end{array}$ & $\mathrm{r}^{2}$ \\
\hline Crotalaria juncea & 0,005294 & 131 & $0,7456^{*}$ \\
C. ocroleuca & 0,004679 & 148 & $0,9620^{*}$ \\
C. spectabilis & 0,008882 & 78 & $0,9639^{*}$ \\
Feijão de porco & 0,008601 & 81 & $0,9970^{*}$ \\
Guandu & 0,005346 & 130 & $0,9516^{*}$ \\
Mucuna cinza & 0,009117 & 76 & $0,9767^{*}$ \\
Mucuna preta & 0,007781 & 89 & $0,9783^{*}$ \\
\hline
\end{tabular}

Em estudos com diferentes culturas de cobertura de potencial para uso agrícola no Recôncavo Baiano, Xavier et al. (2017), verificaram o tempo de meia vida do feijão de porco de 38 dias, enquanto a Crotalaria juncea teve maior resistência a decomposição, chegando aos 99 dias seu tempo de meia vida. Por se tratar de um processo biológico, a dinâmica da decomposição de resíduos vegetais depende, dentre outros fatores da espécie, do volume de produção de biomassa e das condições climáticas como a pluviosidade e temperatura (CARVALHO et al., 2015). Demonstra-se a importância e viabilidade de uso de leguminosas como estratégia de intensificação ecológica para alcançar uma agricultura conservacionista de recursos naturais, especialmente para o solo.

\section{CONCLUSÕES}

As palhadas do feijão guandu e as crotalárias juncea e ocroleuca apresentam as maiores recalcitrâncias, suficientes para proteção da superfície do solo durante 0 período de entressafra do milho verão na região Amazônica.

\section{AGRADECIMENTOS}

Ao CNPq por suporte financeiro na condução do experimento e análise dos tecidos pelo projeto Universal processo 459130/2014-5.

\section{REFERÊNCIAS}

AKER, A. M.; PASSOS, A. M. A.; MARCOLAN, A. L.; SANTOS, F. C.; CIPRIANI, H. N.; VARGAS, L. A. Plantas de cobertura sobre atributos agronômicos da cultura do milho na região Sudoeste da Amazônia. Revista Brasileira de Milho e Sorgo, v. 15, 
n. 3, p. 532-543, 2016. Disponível em: http://dx.doi.org/10.18512/19806477/rbms.v15n3p531-542

AKER, A. M.; PASSOS, A. M. A.; COSTA, R. S. C.; SANTOS, F. C.; LEITE, V. P. D.; MARCOLAN, A. L. Effect of cover crops on physico-chemical attributes of soil in a short-term experiment in the southwestern Amazon region. African Journal of Agricultural Research, v. 12, n. 47, p. 3339-3347, 2017. Disponível em: https://doi.org/10.5897/AJAR2017.12800

ALVARES, C. A.; STAPE, J. L.; SENTELHAS, P. C.; DE MORAES GONÇALVES, J. L.; SPAROVEK, G. Köppen's climate classification map for Brazil. Meteorologische Zeitschrift, v. 22, n. 6, p. 711-728, 2013. Disponível em: http://dx.doi.org/10.1127/0941-2948/2013/0507

BOER, C. A.; ASSIS, R. L.; SILVA, G. P.; BRAZ, A. J. B. P.; BARROSO, A. L. L.; CARGNELUTTI FILHO, A.; PIRES, F. R. Ciclagem de nutrientes por plantas de cobertura na entressafra em um solo de cerrado. Pesquisa Agropecuária Brasileira, v. 42, p. 1269-1276, 2007. Disponível em: http://dx.doi.org/10.1590/S0100-204X2007000900008

CARVALHO, A. M.; COSER, T. R.; REIN, T. A.; DANTAS, R. A.; SILVA, R. R.; SOUZA, K. W. Manejo de plantas de cobertura na floração e na maturação fisiológica e seu efeito na produtividade do milho. Pesquisa Agropecuária Brasileira, v. 50, n. 7, 551-561, 2015. Disponível em: http://dx.doi.org/10.1590/S0100-204X2015000700005

CAVALLI, E.; LANGE, A.; CAVALLI, C.; BEHLING, M. Decomposition and release of nutrients from crop residues on soybean-maize cropping systems. Brazilian Journal of Agricultural Sciences, v. 13, n. 2, p. 1-8, 2018. Disponível em: https://doi.org/10.5039/agraria.v13i2a5527

DARYANTO, S.; FU, B.; WANG, L.; JACINTHE, P. A.; ZHAO, W. Quantitative Synthesis on the Ecosystem Services of Cover Crops. Earth-Science Reviews, v. 185 p. 357-373, 2018. Disponível em: https://doi.org/10.1016/j.earscirev.2018.06.013.

DERPSCH, R.; FRANZLUEBBERS, A. J.; DUIKER, S. W.; REICOSKY, D. C.; KOELLER, K.; FRIEDRICH, T.; STURNY, W. G.; SÁ, J. C. M.; WEISS. K. Why Do We Need to Standardize No-Tillage Research? Soil and Tillage Research, v. 137, p. 16-22, 2014. Disponível em: http://dx.doi.org/10.1016/j.still.2013.10.002

HEINRICHS, R.; VITTI, G. C.; MOREIRA, A.; FIGUEIREDO, P. A. M.; FANCELLI, A. L.; CORAZZA, E. J. Características químicas de solo e rendimento de fitomassa de adubos verdes e de grãos de milho, decorrente do cultivo consorciado. Revista Brasileira de Ciência do Solo, v. 29, n. 1, p. 71-79, 2005. Disponível em: https://dx.doi.org/10.1590/S0100-06832005000100008

LAL, R. Soil carbon sequestration and aggregation by cover cropping. Journal of Soil and Water Conservation, v. 70, n. 6, p. 385-398, 2015. Disponível em: https://dx.doi.org/10.2489/jswc.70.6.329 
MALAVOLTA, E.; VITTI, G. C.; OLIVEIRA, S. A. Avaliação do estado nutricional das plantas - princípios e aplicações. 2. ed. Piracicaba: POTAFOS, 1997. 309 p.

MATATA, P. Z., PASSOS, A. M. A., MASOLWA, L. M., MARCOLAN, A. L., RIBEIRO, R. da S. Incorporation of leguminous cover crops in smallholder cassava-based production system in Western Tanzania. American Journal of Plant Sciences, v. 8, p. 3490-3501, 2017. Disponível em: https://dx.doi.org/10.4236/ajps.2017.813235

MBUTHIA, L. W.; ACOSTA-MARTÍNEZ, V.; DEBRUYN, J.; SCHAEFFER, S.; TYLER, D.; ODOI, E.; MPHESHEA, M.; WALKER, F.; EASH, N. Long term tillage, cover crop, and fertilization effects on microbial community structure, activity: Implications for soil quality. Soil Biology and Biochemistry, v. 89, p. 24-34, 2015. Disponível em: https://dx.doi.org/10.1016/j.soilbio.2015.06.016

MIRANDA, S. C.; MATA, C. R.; FONSECA, K. S.; CARVALHO, P. S. Apontamentos sobre mudanças climáticas na agricultura Brasileira. Revista Enciclopédia Biosfera, v.15, n. 27, p. 95-106, 2018.Disponível em: https://dx.doi.org/10.18677/EnciBio_2018A9

PEREIRA, A. P.; SCHOFFEL, A.; KOEFENDER, J.; CAMERA, J. N.; GOLLE, D. P.; HORN, R. C. Ciclagem de nutrientes por plantas de cobertura de verão. Revista de Ciências Agrárias, v. 40, n. 4, p. 799-807, 2017. Disponível em: http://dx.doi.org/10.19084/RCA17065

SANTOS, P.F. e WHILFORD, W.G. The effects of microarthropods on litter decomposition in a chihuazhuan ecosystem. Ecology, 62:654-669, 1981. Disponível em: https://doi.org/10.2307/1937733

SANTOS, P. A. SILVA, A. F.; CARVALHO, M. A. C.; CAIONE, G. Adubos verdes e adubação nitrogenada em cobertura no cultivo do milho. Revista Brasileira de Milho e Sorgo, v. 9, n. 2, p. 123-134, 2010. Disponível em: http://dx.doi.org/10.18512/1980-6477/rbms.v9n2p123-134

TEIXEIRA, C. M.; CARVALHO, G. J.; SILVA, C. A.; ANDRADE, M. J. B.; PEREIRA, J. M. Liberação de macronutrientes das palhadas de milheto solteiro e consorciado com feijão-de-porco sob cultivo de feijão. Revista Brasileira de Ciência do Solo, v. 34, n. 2, p. 497-506, 2010. Disponível em: https://dx.doi.org/10.1590/S010006832010000200023

THOMAS, R. J.; ASAKAWA, N. M. Decomposition of leaf litter from tropical forage grasses and legumes. Soil Biology and Biochemistry, v. 25, n. 10, p. 1351-1361, 1993. Disponível em: https://doi.org/10.1016/0038-0717(93)90050-L

TORRES, J. L. R.; PEREIRA, M. G.; JUNIOR RODRIGUES, D. J.; LOSS, A. Production, decomposition of residues and yield of maize and soybeans grown on cover crops. Revista Ciência Agronômica, v. 46, n. 3, p. 460-468, 2015. Disponível em: http://dx.doi.org/10.5935/1806-6690.20150026 
VALADARES, R. V.; DUARTE, R. F.; MENEZES, J. B. C.; FERNANDES, L. A.; SANTOS TUFFI, L. D.; SAMPAIO, R. A.; MOTA, T. C.; ALMEIDA, R. M. Fertilidade do solo e produtividade de milho em sistemas de adubação verde no norte de Minas Gerais. Planta Daninha, v. 30, n. 3, p. 505-516, 2012. Disponível em: https://dx.doi.org/10.1590/S0100-83582012000300006

XAVIER, F. A. S.; OLIVEIRA, J. I. A.; SILVA, M. R. Decomposition and nutrient release dynamics of shoot phytomass of cover crops in the Recôncavo Baiano. Revista Brasileira de Ciência do Solo, v. 41, p. 1-14, 2017. Disponível em: https://dx.doi.org/10.1590/18069657rbcs20160103 